Bettina Ute Tillmann · Hanns-Christian Tillmann

Ulrich Heininger · Jürg Lütschg · Peter Weber

\title{
Acceptance and timeliness of standard vaccination in children with chronic neurological deficits in north-western Switzerland
}

Received: 26 July 2004/ Revised: 15 December 2004/ Accepted: 23 December 2004/Published online: 25 February 2005

(C) Springer-Verlag 2005

\begin{abstract}
There are no special recommendations for basic vaccinations in patients with chronic neurological deficits distinct from the nationwide advocated schedule in Switzerland. Reports describing adverse neurological events possibly related to vaccinations have attracted public attention. It is unclear if patients with chronic neurological deficits are more reluctantly vaccinated compared to healthy children. We therefore investigated the acceptance of vaccinations in such patients and healthy controls in a retrospective case-control study. At the University Children's Hospital, Basel, Switzerland we investigated 100 patients with chronic neurological deficits and 200 age-matched healthy controls regarding the issue of vaccination rates and ages. The total number of administered vaccinations against diphtheria, tetanus, pertussis, polio, Haemophilus influenzae type b (Hib), mumps, measles, rubella and hepatitis $\mathrm{B}$ were significantly lower in patients compared to healthy controls $(P<0.01$ for each of the respective vaccines). Patients had an increased risk to receive the third pertussis, diphtheria, and tetanus vaccinations (relative risks (RR) for late vaccination $1.53,1.53$, and 1.54 respectively, $P<0.01$ for all comparisons), the second $(\mathrm{RR}=1.60, P<0.05)$ and third Hib vaccinations $(\mathrm{RR}=1.52, P<0.05)$, and the third polio vaccination $(\mathrm{RR}=1.43, P<0.05)$ later than controls. Conclusion: Children with chronic neurological deficits received fewer vaccinations than healthy controls. In addition, patients received vaccinations later than healthy children. Hence, it may be assumed that children with chronic neurological deficits are at an increased risk to acquire preventable infections. Therefore, vaccination
\end{abstract}

B. U. Tillmann $(\bowtie) \cdot$ U. Heininger $\cdot$ J. Lütschg $\cdot$ P. Weber Department of Neuropaediatrics,

University Children's Hospital,

P.O. Box, 4005 Basel, Switzerland

E-mail: btillmann@yahoo.com

Tel.: + 41-61-6856565

Fax: +41-61-6856566

H.-C. Tillmann

Biomit Inc., Basel, Switzerland should be promoted as part of the consultation during a routine appointment with the specialist.

Keywords Chronic neurological deficit . Immunisation - Vaccination - Vaccination age · Vaccination rate

Abbreviations DTP: diphtheria, tetanus, pertussis hep B: hepatitis B - Hib: Haemophilus influenzae type B - MMR: mumps, measles and rubella $\cdot \mathrm{RR}$ : relative risk

\section{Introduction}

Vaccinations prevent infections and their complications [5]. Many countries have introduced nationwide vaccination schedules. The acceptance of vaccinations is influenced by a variety of factors. Missed preventive visits, and difficulties in tracking undervaccinated patients play a major role in lacking compliance with recommendations [21]. In addition, children with preexisting medical conditions such as premature birth are vaccinated more reluctantly than full-term born children [22].

There are no special recommendations regarding the standard vaccinations for children with chronic diseases only some specific explanations and precautions are given, for example for pertussis vaccination. Early reports of adverse neurological events following different vaccinations have led to a strong reluctance to vaccinate these patients according to nationwide schedules [2]. However, the reluctance to immunise children with neurological diseases has not been sufficiently documented. Although a controversial issue, available scientific evidence underlines the importance of vaccination in children with chronic neurological deficits [7]. With modern vaccines, serious adverse reactions are extremely rare and the overall benefit by far outweighs possible 
adverse reactions [13, 16]. Nevertheless, practitioners and parents often hesitate to vaccinate children with chronic neurological deficits, possibly due to an unawareness of guidelines or an unwillingness to put them into practice $[8,18,21]$.

We therefore examined children with chronic neurological deficits regarding vaccination rate and the latency of vaccine administration.

\section{Subjects and methods}

The University Children's Hospital of Basel, Switzerland, serves children with neurological diseases in north-western Switzerland (approximately 650,000 inhabitants). Between March 1999 and April 2001, we accumulated vaccination data of 100 patients in our neuropaediatric outpatient clinic. After approval by the local Ethics Committee, patients with various chronic neurological diseases were collected to obtain information on the acceptance of vaccination in this group of patients and to avoid reservation concerning particular neurological diseases. We included patients with chronic neurological deficits at the age of 1 to 16 years. As controls we collected 200 primarily healthy agematched children who presented with an acute illness in our general outpatient clinic. Data of patients and controls were gathered by convenience sampling. The patients and controls were representative of all children in this geographic area. All children were raised in north-western Switzerland. Excluded were children with other chronic diseases or premature birth.

After oral informed consent, parents were asked to present a copy of their children's vaccination certificate. Minimisation of selection bias in the patient group was achieved since $100 \%$ of parents who were asked to participate in fact consented to submit their children's vaccination certificates. The sample of healthy controls was potentially more prone to residual selection bias since primarily those patients were enrolled whose parents had their children's vaccination certificates with them during the outpatient clinics visit.

We restricted our study to those vaccinations suggested by the Swiss committee for immunisation recommendations, namely diphtheria, tetanus, pertussis (DTP), polio, Haemophilus influenzae type b (Hib), mumps, measles, rubella (MMR), and hepatitis B (hep B). While the study was performed, four vaccinations against polio, diphtheria and tetanus, and Hib and three against pertussis were recommended in Switzerland during the first 2 years of life. Recommended vaccination ages were 2, 4, 6, and 15-24 months for polio, DTP and Hib. One vaccination against MMR was recommended between 12 and 24 months of age. Children older than 11 years had to be vaccinated against hep B three times [9] ( $n=66$ in this study). Analyses of vaccinations against $\mathrm{Hib}$ were restricted to patients and respective controls born in 1987 or later $(n=270)$ since the vaccine became available no earlier than 1991.
Children born in 1987 or later were recommended to catch up on Hib vaccinations.

\section{Statistical evaluation}

Primary evaluation parameters were vaccination rate and age of the entire patient population compared to age-matched healthy controls. Triplets were built consisting of one patient and two age-matched controls. Simple rates were calculated for all vaccinations. Vaccination rates were evaluated within the triplets with the patient value being compared to the mean of the two respective control values. The Wilcoxon signed rank test was used for symmetric data (sign test for asymmetric data) to determine whether average within triplet differences in vaccination parameters between patients and controls were statistically different from 0 . All tests were performed two-sided at a significance level of 5\%.

To determine vaccination age we counted the days from the date of birth to the date of each performed vaccination. Survival-analytical methods were used to compare vaccination ages. Vaccinations were considered as events. In the case of missing vaccinations, time to vaccination was censored by the subject's age at the day of our examination. The influence of being a patient on time to vaccination was then assessed using Cox regression models with an individual baseline hazard function estimate for each triplet.

A subset of patients was chosen according to age at the time of diagnosis (congenital plus diagnosis within the first 6 months of life, $n=66$ ) to investigate vaccination patterns in more detail.

We also examined if patients and controls were vaccinated within a timeframe compatible with the published recommendations. Since only the first three vaccinations against DTP, Hib, polio, and the first vaccination against MMR are recommended to be administered at a particular age, we restricted our analysis to these vaccinations. We subtracted the recommended vaccination age from the child's age at vaccination and tested whether the population mean of the resulting number of days was significantly different from zero using $t$-tests for unpaired samples. This was done separately for patients and healthy controls and in both groups for only those subjects who received the respective vaccinations. Analyses account for all changes of recommended vaccination ages in Switzerland between 1986 and 2000.

\section{Results}

Patient characteristics

Patients were between 1 and 16 years old (Table 1). A total of 55 patients had congenital abnormalities and 45 acquired a chronic neurological deficit during the first 4 years of life. In these patients, median age at diagnosis 
Table 1 Demographic data of patients with chronic neurological diseases and age-matched healthy controls

\begin{tabular}{|c|c|c|}
\hline & Patients $(n=100)$ & Controls $(n=200)$ \\
\hline $\begin{array}{l}\text { Gender } \\
\text { (male/ female) }\end{array}$ & $59 \% / 41 \%$ & $55 \% / 45 \%$ \\
\hline Ethnicity & $\begin{array}{l}\text { Switzerland }(79 \%) \\
\text { Turkey }(15 \%) \\
\text { Sri Lanka }(2 \%) \\
\text { Yugoslavia }(2 \%) \\
\text { Italy }(1 \%) \\
\text { Germany }(1 \%)\end{array}$ & $\begin{array}{l}\text { Switzerland }(83 \%) \\
\text { Turkey }(7 \%) \\
\text { Sri Lanka }(3.5 \%) \\
\text { Yugoslavia }(3 \%) \\
\text { Italy }(2.5 \%) \\
\text { Germany }(0.5 \%) \\
\text { Togo }(0.5 \%)\end{array}$ \\
\hline Main diagnoses & $\begin{array}{l}\text { Epilepsy }(39 \%) \\
\text { Cerebral palsy }(24 \%) \\
\text { Severe developmental } \\
\text { delay }(17 \%) \\
\text { Recurrent complicated } \\
\text { febrile seizures }(10 \%) \\
\text { Neurodegenerative, } \\
\text { metabolic diseases }(6 \%) \\
\text { Myopathies }(4 \%)\end{array}$ & Not applicable \\
\hline $\begin{array}{c}\text { Mean age (years } \pm \\
\text { standard error) }\end{array}$ & $6.7 \pm 0.4$ & $6.7 \pm 0.3$ \\
\hline
\end{tabular}

was 1.4 years (range 20 days -4.0 years). The most frequent deficits were epilepsy (39\%), cerebral palsy (11\%), degenerative and metabolic affections of the central nervous system $(10 \%)$, and recurrent febrile seizures $(10 \%)$.

\section{Vaccination rate}

Patients with congenital neurological deficits or a diagnosis within the first 6 months of life $(n=66)$ had substantially lower vaccination rates than age-matched healthy controls $(n=132)$. Over $88 \%$ of patients and healthy controls received the first three doses of polio, diphtheria and tetanus vaccine. Pertussis, Hib, MMR, and hep B vaccines were less frequently administered. Except for the first dose of polio and T, patients had lower vaccination rates than healthy controls. This was statistically significant for the third doses of diphtheria and tetanus. All four doses of pertussis vaccine were administered to patients less often than to controls with a statistical significance for the first three doses (Table 2).

\section{Vaccination age}

Children with chronic neurological deficits were at an increased risk for being vaccinated against DTP, polio, and $\mathrm{Hib}$ later than age-matched healthy controls (Fig. 1). Patients had an increased risk of $36 \%$ and $31 \%$ of receiving the first and second dose of pertussis vaccine later than controls. A $54 \%$ increased risk of receiving the third vaccination late was statistically significant $(P<0.01)$. A very similar picture evolved for the comparison between patients and controls for
Table 2 Vaccination rates ( $\%$ ) of patients with congenital neurological diseases or diagnosis within the first 6 months of life compared to age-matched healthy controls

\begin{tabular}{lllll}
\hline Vaccination & Dose 1 & Dose 2 & Dose 3 & Dose 4 \\
\hline Diphtheria & & & & \\
Patients $(n=66)$ & 97 & 96 & $89^{*}$ & 70 \\
$\quad$ Controls $(n=132)$ & 99 & 99 & 97 & 78 \\
Tetanus & & & & \\
$\quad$ Patients $(n=66)$ & 99 & 97 & $91^{*}$ & 68 \\
$\quad$ Controls $(n=132)$ & 99 & 99 & 98 & 79 \\
Pertussis & & & & \\
$\quad$ Patients $(n=66)$ & $86^{*}$ & $83^{*}$ & $71^{* *}$ & 36 \\
$\quad$ Controls $(n=132)$ & 95 & 92 & 89 & 49 \\
Polio & & & & \\
$\quad$ Patients $(n=66)$ & 99 & 97 & 88 & 70 \\
$\quad$ Controls $(n=132)$ & 99 & 99 & 96 & 78 \\
Hib & & & & \\
$\quad$ Patients $(n=58)$ & 92 & 80 & 69 & 23 \\
$\quad$ Controls $(n=116)$ & 94 & 88 & 82 & 35 \\
MMR & & & & \\
Patients $(n=66)$ & 70 & 8 & & \\
$\quad$ Controls $(n=132)$ & 79 & 14 & & \\
Hep B & & & \\
Patients $(n=12)$ & 8 & 8 & 0 & \\
$\quad$ Controls $(n=24)$ & 16 & 16 & 12 & \\
\hline
\end{tabular}

* $P<0.05$ compared to healthy controls

** $P<0.01$ compared to healthy controls

vaccinations against diphtheria, tetanus and polio. In addition, patients were at a $60 \%$ increased risk $(P=0.01)$ to receive the second, and at a $52 \%$ increased risk $(P<0.05)$ to receive the third dose of $\mathrm{Hib}$ vaccine later than controls. For MMR and hep B vaccinations, patients had no statistically significant increased risk to receive the dose delayed compared to healthy controls (Fig. 1).

\section{Compliance with vaccination recommendations}

Patients and healthy controls received the analysed vaccinations later than recommended by official Swiss guidelines. The first vaccinations against DTP, and polio were administered with approximately 3 months delay. The first Hib and the MMR vaccinations were also administered with latency compared to the recommended vaccination ages. In patients, the first vaccination against $\mathrm{Hib}$ was given $115 \pm 59$ days and the vaccination against MMR $56 \pm 24$ days later than recommended $(P<0.05)$. In healthy children, the respective vaccinations were administered $38 \pm 5$ and $52 \pm 10$ days later than recommended $(P<0.01)$. The second and third vaccinations against DTP, polio, and Hib were given with a delay of 89 to 188 days in patients and 40 to 155 days in healthy controls. In most instances these differences from the recommended vaccination dates were statistically significant. As a generally applicable example of vaccination coverage we display an inverse KaplanMeier plot [15] for the third dose of pertussis vaccine (Fig. 2). 
Fig. 1 Vaccination age/risk ratio for late vaccination in patients with chronic neurological disease $(n=100)$ compared to age-matched healthy controls $(n=200)$. All risk ratios are presented as point estimates ( solid diamonds) with $95 \%$ confidence intervals in comparison to controls with the predefined control value $=1$. ** $P<0.01, * P<0.05$, compared to healthy controls
Diphtheria 1

Diphtheria 2

Diphtheria 3

Diphtheria 4

Diphtheria max

Tetanus 1

Tetanus 2

Tetanus 3

Tetanus 4

Tetanus max

Pertussis 1

Pertussis 2

Pertussis 3

$* *$

Pertussis 4

Polio 1

Polio 2

Polio 3

$*$

Polio 4

Polio max

Hib 1

Hib 2

Hib 3

*

$*$

Hib 4

MMR 1

MMR 2

Hep b 1

Hep b 2

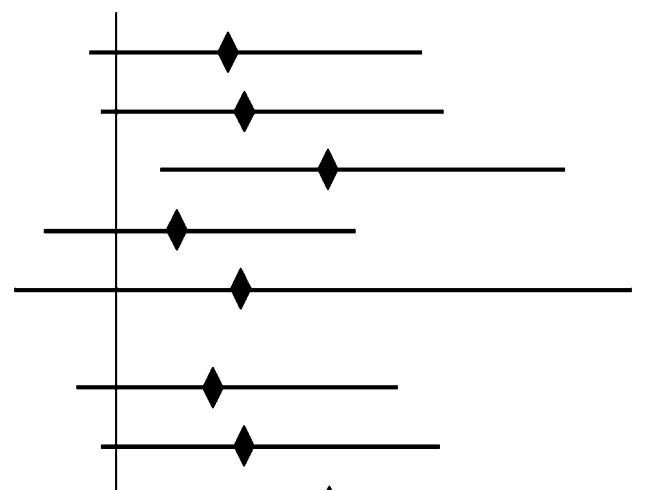

$* *$

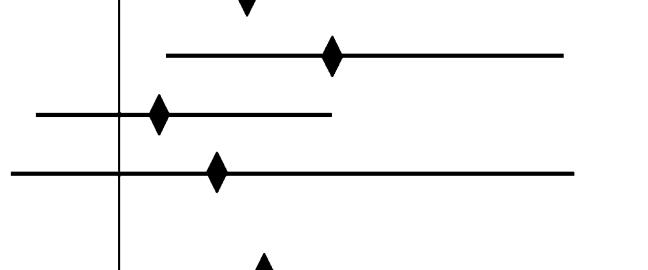

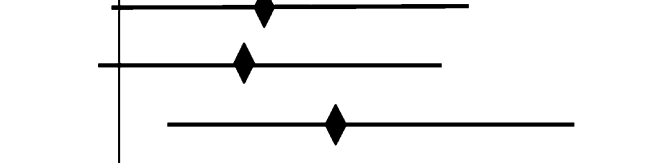
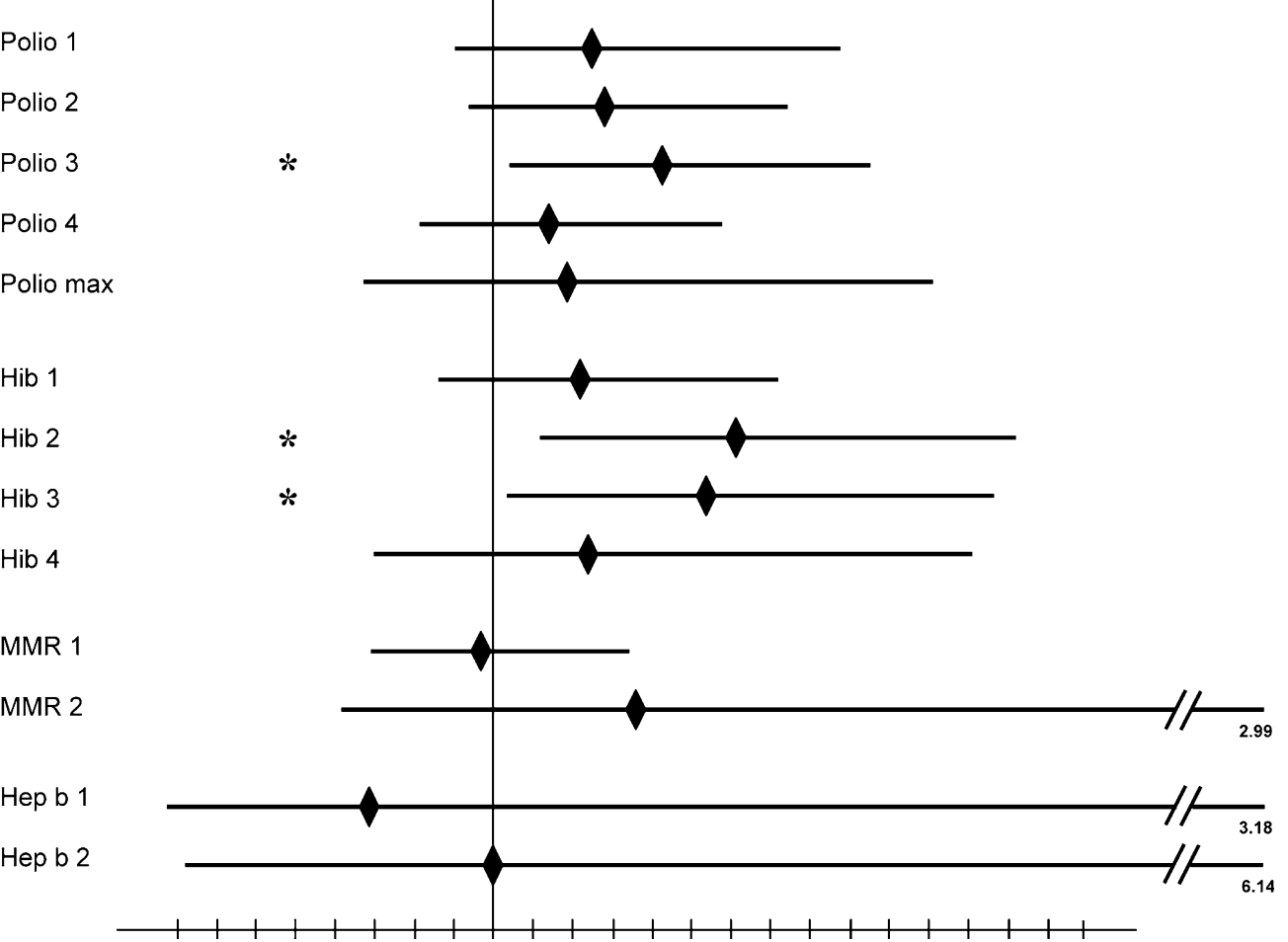

\section{Discussion}

Vaccines against hep B, Hib, MMR, and especially pertussis have been suspected to cause severe illness and in particular neurological diseases [2, 6, 11, 17, 19]. Large-scale epidemiological studies have failed to confirm these allegations $[7,20]$.
Our data express a clear warning sign: patients with underlying chronic neurological deficits receive fewer DTP, polio, Hib, MMR, and hep B vaccinations compared to healthy controls (Table 2). This finding may partly be explained by a study investigating paediatric and family residents' intention to vaccinate children with neurological diseases. Physicians reported a lower likelihood to vaccinate children with a neurological condi- 
tion $[4,14]$. The authors support an intensified education of residents regarding vaccination issues.

Febrile seizures have been reported after receipt of DTP (whole cell) or MMR vaccine but there was no association with long-term side-effects [1]. The modern acellular compared to whole cell pertussis vaccines induce less side-effects $[10,12]$. Nevertheless, children in our study received fewer doses of pertussis than diphtheria, tetanus, and polio vaccine. A low vaccination rate against pertussis may be due to an underestimation of potentially deleterious sequelae following Bordetella pertussis infection [3]. We speculate that a historic scepticism against pertussis vaccination originating from the era of whole cell pertussis vaccines still influences the acceptance of modern vaccines [17]. Patients with chronic neurological deficits and especially children with congenital neurological deficits or a diagnosis within the first 6 months of life received fewer vaccinations than controls (Table 2). This reflects an additional risk to acquire $B$. pertussis infections. However, it has to be kept in mind that the older children in our study population were initially vaccinated with whole cell pertussis vaccine in the early 1990's.

Our results on vaccination ages further corroborate the above assumptions. Patients were at an approximately $30 \%$ to $50 \%$ increased risk to receive pertussis vaccinations later than controls (Fig. 1). For presumably similar reasons, Hib vaccinations were administered less often than other vaccinations in the entire examined population. It has to be considered that since 1995, monovalent Hib vaccines (given twice in the first and once in the second year of life) have been gradually substituted by DTP-Hib combination vaccines (four dose schedule, three doses in the 1st year of life). Additionally, children born before 1991 were recommended to catch up on all Hib doses. Furthermore, a particularly high risk for late vaccination was apparent for the second and third Hib vaccination (Fig. 1).
The finding that vaccination rates against MMR were similarly low among patients and healthy controls is in line with earlier results for the whole of Switzerland [3].

Low vaccination rates against hep B in patients and controls, recommended at the age of 11 years onwards, may be due to the recent introduction in 1997 of this vaccine into nationwide recommendations. Nevertheless, rates for the respective doses between $16 \%$ and $0 \%$ (Table 2) are alarming, particularly for chronically disabled children who are more likely to be in contact with hep B. Vaccination campaigns have obviously not had the desired effect as yet since there has been no measurable increase in vaccination rates compared to 1998 [3]. Therefore, we strongly advocate further attempts to positively change hep B vaccination acceptance.

Another aspect with public health impact is highlighted by our data on the conformity of vaccination dates with the recommended vaccination dates. All examined vaccinations were given later than promoted by guidelines. We were alerted by the fact that many vaccinations were administered as late as 4 to 6 months after the recommended vaccination age in both groups. This was particularly obvious for pertussis (Fig. 2). During this considerable period, children have no adequate protection against the respective diseases. Since all children were affected by this reluctance to vaccinate according to guidelines, we suggest an energetic effort by public health agencies aimed at physicians and parents to emphasise the importance of timely vaccinations.

In conclusion, our data show that children with chronic neurological deficits in north-western Switzerland received less vaccinations and were vaccinated later than age-matched healthy controls. This may entail an increased risk for community-acquired infections. Today, vaccinations can be considered safe but the indication to vaccinate should be determined on an individual basis. The effectiveness of vaccination is dependent on the personal engagement of the primary
Fig. 2 Coverage of pertussis vaccination (dose 3 ) in children with chronic neurological deficits $(n=100)$ compared to age-matched healthy controls $(n=200)$ in north-western Switzerland. The right side of the shaded area marks the nationally recommended ageperiod for vaccination 3 (at the age of 5 months)

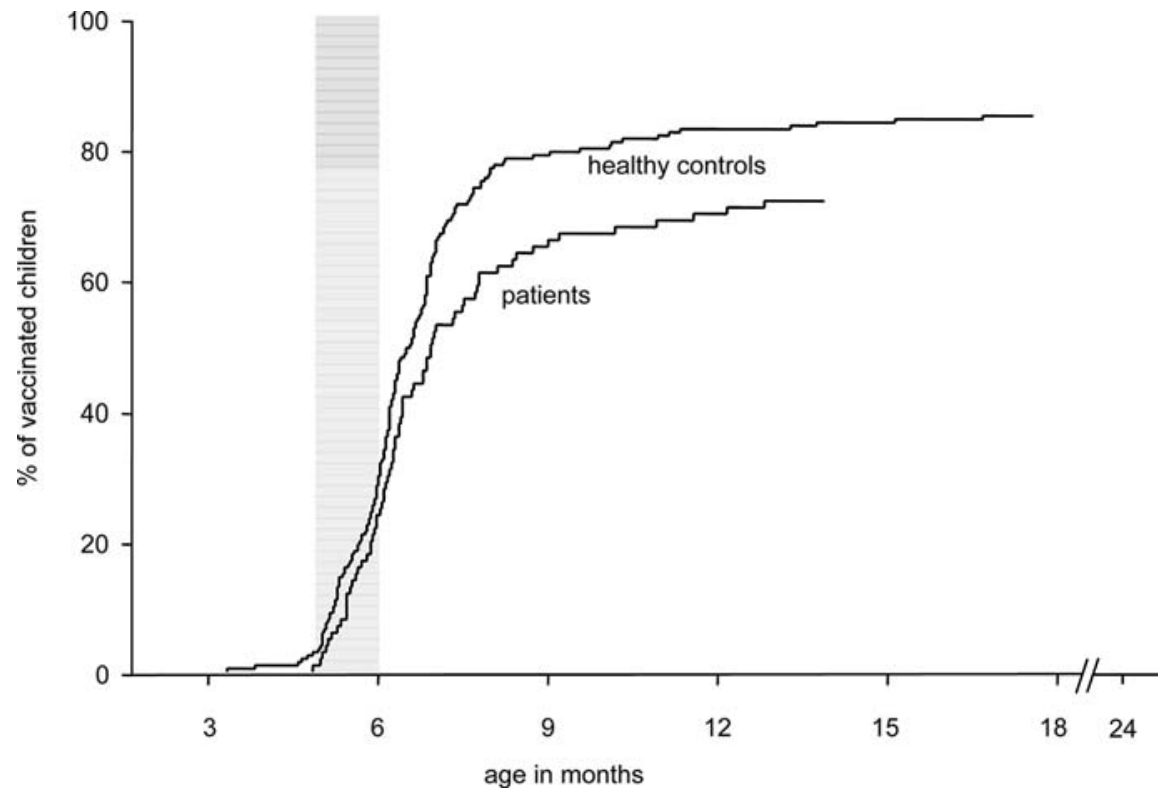


care provider [23] whose role may be taken over by a specialist in children with chronic diseases. Since specialists sometimes tend to neglect general patient care aspects such as vaccinations during an appointment, it has to be stressed that they are obliged to recommend or even provide vaccinations.

Acknowledgements This study could not have been performed without the help of many colleagues and nurses of the University Children's Hospital in Basel, Switzerland. We thank each of them for their contribution, especially R. Bubl, U. Blauenstein and V. Grote. We thank C. Schindler, Institute of Social and Preventive Medicine, University of Basel, Switzerland, for expert statistical advice.

\section{References}

1. Barlow WE, Davis RL, Glasser JW, Rhodes PH, Thompson RS, Mullooly JP et al (2001) Centers for Disease Control and Prevention Vaccine Safety Datalink Working Group: the risk of seizures after receipt of whole-cell pertussis or measles, mumps, and rubella vaccine. N Engl J Med 345: 656-661

2. Brody M, Sorley RG (1947) Neurologic complications following administration of pertussis vaccine. NY State J Med 47: $1016-1017$

3. Bundesamt für Gesundheit der Schweiz (Swiss Federal Health Bureau) (1999) Repräsentative Erhebung zur Durchimpfung in der Schweiz 1998. Bulletin 20: 356-361

4. Campbell JR, Szylagyi PG, Rodewald LE, Winter NL, Humiston SG, Roghmann KJ (1994) Intent to immunize among pediatric and family residents. Arch Pediatr Adolesc Med 148: 926-929

5. Center for Disease Control and Prevention (2001) National, state, and urban area vaccination coverage levels among children age 19-35 months - United States 2000. JAMA 286: 1169 1170

6. Cherry JD (1990) Pertussis vaccine encephalopathy: it is time to recognize it as the myth that it is. JAMA 263: 1679-1680

7. Committee on Infectious Diseases (1991) The relationship between pertussis vaccine and brain damage: reassessment. Pediatrics 88: 397-400

8. Davis RL, Rubanowice D, Shinefield HR, Lewis N, Gu D, Black SB et al (1999) Immunization levels among premature and low birth-weight infants and risk factors for delayed up-todate immunization status. JAMA 282: 547-553
9. Desgrandchamps D (1998) Routine vaccination schedule in childhood. Ther Umsch 55: 8-12

10. Fenichel GM (1999) Assessment: neurologic risk of immunization: report of the Therapeutics and Technology Assessment Subcommittee of the American Academy of Neurology. Neurology 52: 1546-1552

11. Grand MG, Wyll SA, Gehlbach SH, Landrigan PJ, Judelsohn RG, Zendel SA et al (1972) Clinical reactions following rubella vaccination. A prospective analysis of joint, muscular, and neuritic symptoms. JAMA 220: 1569-1572

12. Heininger U (2001) Recent progress in clinical and basic pertussis research. Eur J Pediatr 160: 203-213

13. Howson CP, Fineberg HV (1992) The ricochet of magic bullets: summary of the Institute of Medicine Report, adverse effects of pertussis and rubella vaccines. Pediatrics 89: $318-324$

14. Kempe A, Steiner JF, Renfrew BL, Lowery E, Haas K, Berman $\mathrm{S}$ (2001) How much does a regional immunization registry increase documented immunization rates at primary care sites in rural Colorado? Ambul Pediatr 1: 213-216

15. Lauberau B, Hermann M, Schmitt HJ, Weil J, von Kries R (2002) Detection of delayed vaccinations: a new approach to visualize vaccine uptake. Epidemiol Infect 128: 185-92

16. MacKay IR, Rosen FS (2001) Vaccines and vaccination. N Engl J Med 345: 1042-10537

17. Madsen T (1933) Vaccination against whooping cough. JAMA 101: $187-188$

18. Meszaros JR, Asch DA, Baron J, Hershey JC, Kunreuther H, Schwartz-Buzaglo J (1996) Cognitive processes and the decisions of some parents to forego pertussis vaccination for their children. J Clin Epidemiol 49: 697-703

19. OvetchkineP(2001) Secondary effects of vaccinations. Arch Pediatr 8: $316-320$

20. Patja A, Davidkin I, Kurki T, Kallio MJ, Valle M, Peltola H (2000) Serious adverse events after measles-mumps-rubella vaccination during a fourteen-year prospective follow-up. Pediatr Infect Dis J 19: 1127-1134

21. Szylagyi PG, Rodewald LE, Humiston SG, Hager J, Roghmann KJ, Doane C et al (1994) Immunization practices of pediatricians and family physicians in the United States. Pediatrics 94: 517-523

22. Tillmann BU, Tillmann HC, Nars PW, WeberP(2001) Vaccination rate and age of premature infants $<1500 \mathrm{~g}$ in northwestern Switzerland. Acta Paediatr 90: 1421-1426

23. Vivier PM, Alario AJ, Peter G, Leddy T, Simon P, Mor V (2001) An analysis of the immunization status of preschool children enrolled in a statewide Medicaid managed care program. J Pediatr 139: 624-629 